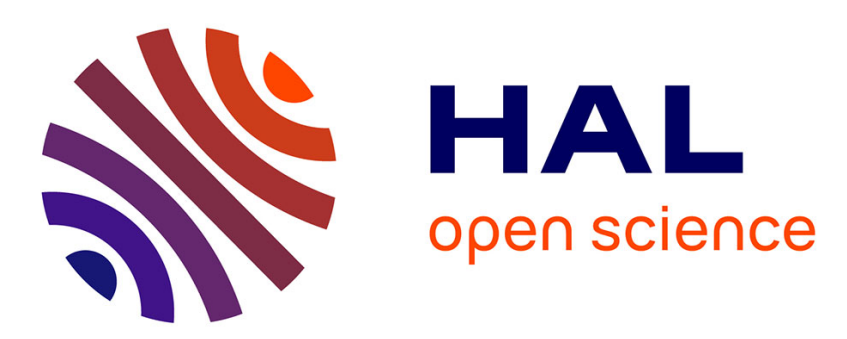

\title{
Subsampling and homogenization to investigate variability of composite material mechanical properties
}

Pierre Pineau, Frédéric Dau

\section{To cite this version:}

Pierre Pineau, Frédéric Dau. Subsampling and homogenization to investigate variability of composite material mechanical properties. Computer Methods in Applied Mechanics and Engineering, 2012, 241-244, pp.238-245. 10.1016/j.cma.2012.06.003 . hal-01006673

\section{HAL Id: hal-01006673 https://hal.science/hal-01006673}

Submitted on 24 Jun 2014

HAL is a multi-disciplinary open access archive for the deposit and dissemination of scientific research documents, whether they are published or not. The documents may come from teaching and research institutions in France or abroad, or from public or private research centers.
L'archive ouverte pluridisciplinaire HAL, est destinée au dépôt et à la diffusion de documents scientifiques de niveau recherche, publiés ou non, émanant des établissements d'enseignement et de recherche français ou étrangers, des laboratoires publics ou privés. 


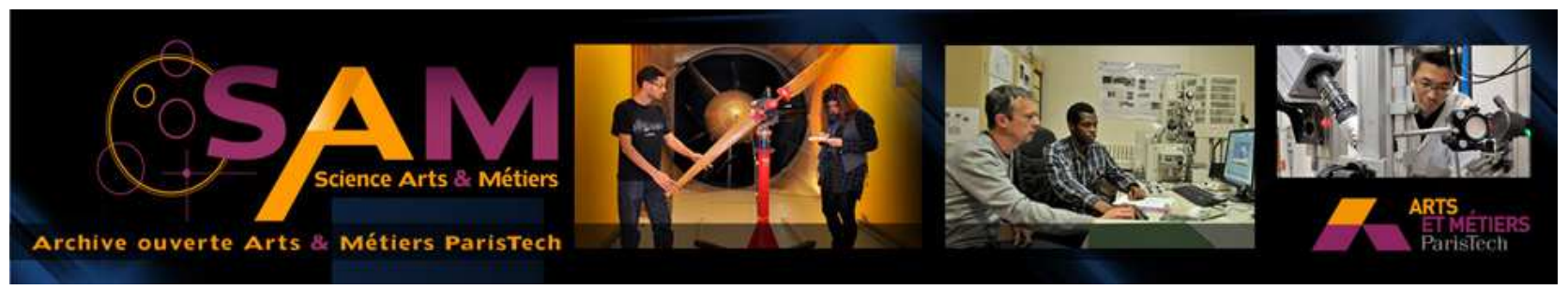

\section{Science Arts \& Métiers (SAM)}

is an open access repository that collects the work of Arts et Métiers ParisTech researchers and makes it freely available over the web where possible.

This is an author-deposited version published in: http://sam.ensam.eu

Handle ID: .http://hdl.handle.net/10985/8260

\section{To cite this version :}

Pierre PINEAU, Frédéric DAU - Subsampling and homogenization to investigate variability of composite material mechanical properties - Computer Methods in Applied Mechanics and Engineering - Vol. 241-244, p.238-245 - 2012 


\title{
Subsampling and homogenization to investigate variability of composite material mechanical properties
}

\author{
Pierre Pineau ${ }^{\mathrm{a}, *}$, Frédéric Dau ${ }^{\mathrm{b}}$ \\ a LaMCoS - UMR 5259 (CNRS - INSA - Université de Lyon), INSA, bât. Joseph Jaquard, 27 av. Jean Capelle, 69621 Villeurbanne Cedex, France \\ ${ }^{\mathrm{b}}$ I2M, dpt DuMAS - UMR 5295 (CNRS, Université Bordeaux 1, Arts et Métiers ParisTech), Esplanade des Arts et Métiers, 33405 Talence Cedex, France
}

Keywords:

Structural composites

Elastic properties

Finite Element Analysis (FEA)

Multiscale modeling

Probabilistic methods

\begin{abstract}
A B S T R A C T
This paper presents an innovative homogenization sampling technique applied to multiscale modelling of composite materials. The goal is to build efficiently statistical variability of mechanical properties at mesoscopic scale from the heterogeneous media analysis at microscopic scale. It is applied to the transverse elastic properties of a unidirectional Long Fibres Reinforced Composite (LFRC). A large representative part of the ply - the cell - is modelled from a micrography and studied at microscopic scale with the Finite Element Analysis (FEA) under 2D plane strain hypothesis. The study consists in estimating the effective elastic properties of subcells, subparts of the previous cell, thanks to a specifically developed numerical procedure.

A unique calculation is computed on the entire ply reduced to three basic loading cases is applied to the cell. Subsamples taken into the simulation cell are homogenized at post-processing level of strain and stress fields. A standard mechanics approach was considered. Various subsampling schemes are performed with various size and spatial distribution to generate variability functions of effective elastic properties at mesoscopic scale. A statistical inference is highlighted: the variability parameters vary with the way of sampling. Dispersion functions are finally obtained and discussed.
\end{abstract}

\section{Introduction}

\subsection{Working context}

The objective of this work is to develop an efficient way to obtain and characterise the variability of the mechanical properties at intermidiate scale between microscopic and mesoscopic scale taking into account the variability of the mechanical properties at od constituents; the microscopic to mesoscopic variability transport, see Fig. 1(c), is particularly adressed in this paper. As explored in [5], the approach can be transposed from the mesoscale to the macroscale. Then, knowing the variability at different scale through distributions functions, the authors are furtherly interested by performing reliable analysis. The present paper focuses on investigations concerning linear elastic properties variability with the view to work laterly on stresses, damage, failure singularities and non linear behaviour. The starting point is a morphological analysis realized on a micrography of a unidirectional long fibre ply microstructure extracted from a stratified LFRC. A FE mesh of the microstructure representative of a real material configuration is then realised. A FEA is applied to compute strongly heterogeneous strain and stress fields.

\footnotetext{
* Corresponding author. Tel.: +33 472436 411; fax: +33 472438913 .

E-mail addresses: pierre.pineau@insa-lyon.fr(P.Pineau),f.dau@i2m.u-bordeaux1.fr (F. Dau)
}

Many authors developed homogenization techniques to get the effective properties of heterogeneous materials. Most of the time, the Representative Volume Element (RVE) concept is considered $[15,10,11]$ : studying a large enough part of the material gives a global estimation of a given effective property. In [1], homogenization is performed under RVE size and depicts variability of behaviour. To get suitable elastic properties, two criteria have to be verified: the inter scales energy equivalence depicted by the so-called Hill lemma $[7,17]$ and the convergence of effective properties depending on the size of homogenized volume. The energy criterion is verified for smaller volumes than these needed for properties convergence [19]. Other authors [18,4] worked on cells smaller that the RVE - that are called here subcells - and developed statistical approaches to obtain global effective properties from the effective properties of a large population of subcells.

The RVE or the statistical subcells are usually exploited with FEM models $[8,3,16]$. Various homogenization techniques are numerically performed to obtain bounds or statistical moments of effective properties, at least average values. Kinematic Uniform Boundary Conditions (KUBC), Stress Uniform Boundary Conditions (SUBC) or Mixed Boundary Conditions (MBC) are the easiest to compute. Periodic Boundary Conditions give a better accuracy on the effective properties and can advantageously be applied regardless on the geometry of the medium but are not trivial to implement in a FEA tool. All boundary conditions applied in works cited before lead to energetically accurate estimation of effective properties; in 


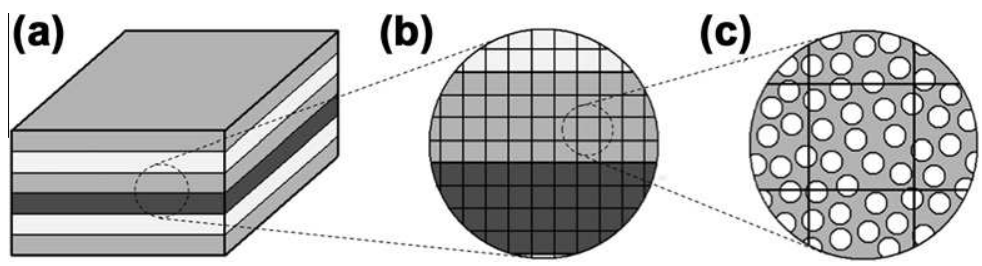

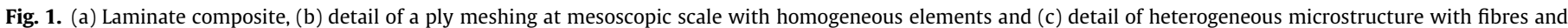
matrix.

case of non periodic loading conditions, some precautions have to be taken to ensure the estimation validity.

In order to get the variability of elastic properties at mesoscopic scale for a scale length inferior to the RVE size, a statistical approach on subcells is realized. One FEM simulation is usually performed on each subcell [4]. It leads to unreasonable computing times as huge number of subcells is required to assess the first statistical moments. To reduce the amount of FEA calculations, a sampling procedure performed at post-processing level and including standard homogenization on non-periodic geometry is developed in this work. It is inspired from morphological analysis of random heterogeneous media [12,13] and has been performed in [1] with only regular spatial repartition. Distribution functions of effective elastic properties are obtained and discussed depending on the subsampling scheme for a statistical inference issue, historically explored in geostatistics [14].

\subsection{Investigation method}

The starting point is a micrography of a LFRC transverse section ply. Under invariance hypothethis in fibre direction, the study can be restricted to a 2D plane strain analysis. On this micrography, a random organisation of carbon fibres in epoxy matrix is observed. This micrography is considered representative of the material microstructure. It is transformed into a FE mesh which defines the so-called cell.

The final point is to obtain the statistical distributions describing the dispersion of the effective elastic properties of smaller zones taken in the ply, the subcells, selected through a subsampling procedure. Their size correspond to a predefined discretization of the ply and consequently the cell, for example a mesoscopic FE mesh as seen Fig. 1(c).

The micrography is digitized and a threshold is peformed on grey levels to generate a FEM mesh of the microstructure. Only three basic loading cases are performed under 2D plane strain hypothesis for the whole work, Section 2. The subsampling procedure is achieved at post processor level on FE strain and stress computed before. A numerical nonperiodic homogenization is performed on subcells following random or organized sampling schemes, Section 3.

Subsampling presents many advantages compared to studies involving virtual material and Monte Carlo procedure. The potential number of subcells to be characterised is wide and directly linked to the resolution of the starting micrography. Moreover subcells don't need to be virtually generated as they are various parts of the meshed simulation cell: they are morphologically representative of the microstructure of the ply.

\section{Hill-Mandell numerical homogenization on nonperiodic geometry}

\subsection{Standard mechanics approach}

The following formalism describes the standard mechanics to obtain effective properties of an heterogeneous medium. It is inspired from $[10,9]$.
Considering a subcell $\Omega$ taken in the heterogeneous medium, an average operator $\langle\cdot\rangle^{\Omega}$ or $\cdot$ is applied to any spatial function defined on the domain, most particularly to stress and strain fields in mechanical applications. Using the Voigt indices contraction convention, stress $\sigma$ and strain $\epsilon$ are written as column matrices and behaviour operators $C$ or $S$ as square matrices. The goal is to estimate the effective stiffness $C_{\text {eff }}$ defined Eq. (1).

$\bar{\sigma}^{\Omega}=C_{e f f}^{\Omega} \bar{\epsilon}^{\Omega}$

The strain and stress fields are decomposed in their average part and a second part called here the perturbation field; for example strain field is decomposed Eq. (2).

$\epsilon=\bar{\epsilon}^{\Omega}+\tilde{\epsilon}^{\Omega}$

A microstructure localization tensor $M^{\Omega}$ is introduced Eq. (3) to link the perturbation and average strain fields, adapted from literature [9].

$\tilde{\epsilon}^{\Omega}=M^{\Omega} \bar{\epsilon}^{\Omega}$

The localization tensor is injected in the local behaviour equation. The average of behaviour relation gives an explicit expression of the effective stiffness explained Eq. (4).

$C_{e f f}^{\Omega}=\left\langle C+C M^{\Omega}\right\rangle^{\Omega}$

It is highlighted that the localization tensor can present minor symmetries $M_{i j k l}=M_{j i k l}=M_{i j l k}$ in agreement with Voigt convention, but not generally major symmetry $M_{i j k l}=M_{k l i j}$. Consequently, the symmetry of behaviour tensor $C_{\text {eff }}^{\Omega}$ can be affected as evoked in [9], in conflict with the definition of thermodynamic potentials in the elastic behaviour.

The same method being applied to stress fields, defining the concentration operator $L^{\Omega}$, the effective compliance $S_{\text {eff }}^{\Omega}$ is explained Eq. (5).

$S_{\text {eff }}^{\Omega}=\left\langle S+S L^{\Omega}\right\rangle^{\Omega}$

The effective properties tensor could be directly obtained from average strain and stress fields, inverting the local behaviour relation. But the calculation of localization and concentration tensors allows to compute the effective properties through various ways and to verify the relevance of numerical results.

\subsection{Energy balance: Hill lemma}

The advantage of the general approach is that it can be applied regardless of any hypothesis on fields' properties or scale decoupling, the effective operators depending on the average values of fields. Both of them should conform to the scale energy balance characterized by the Hill lemma explained in Eq. (6). Using Voigt convention, the elastic strain energy volume density is half the scalar product of strain and stress columns. The reference to cell $\Omega$ is not used laterly to simplify mathematical notations.

$\langle\sigma \cdot \epsilon\rangle=\bar{\sigma} \cdot \bar{\epsilon}$

$\because$ defining the scalar product. 
In our case, it is easily proved that the averages of perturbation fields are equal to zero. The average energy on the cell is then developed, simplified and expressed Eq. (7).

$\langle\sigma \cdot \epsilon\rangle=\bar{\sigma} \cdot \bar{\epsilon}+\langle\tilde{\sigma} \cdot \tilde{\epsilon}\rangle$

In standard homogenization techniques, the average of perturbation elastic strain energy is null, given the uniform or periodic boundary conditions or fields analytical conformation. In our case, there are no guaranties that it is true. The subsampling conditions are investigated to verify the energy balance. An energy error indicator is defined Eq. (8). It is simply the ratio between perturbations product and averages product. The energy balance is considered verified when the error energy is small enougth.

$e R=\frac{\langle\tilde{\sigma} \cdot \tilde{\epsilon}\rangle}{\bar{\sigma} \cdot \bar{\epsilon}}$

\subsection{Numerical procedure}

Basic triangle elements with linear interpolation are used for the FEA. They are not very accurate for strain and stress field calculation but are consequently the easiest to develop our post-processing tools. A mesh refinement naturally increases the quality of $\mathrm{FE}$ analysis without penalizing the numerical procedure. The FE simulations are realized under $\left(\overrightarrow{e_{1}}, \overrightarrow{e_{2}}\right)$ plane strain hypothesis which suggest geometry invariance along the fibres direction. Strain and stress tensors are $3 \times 1$ columns and stiffness, compliance, localization and concentration tensors $3 \times 3$ matrices. For example, the strain is given Eq. (9).

$\epsilon=\left[\begin{array}{l}\epsilon_{11} \\ \epsilon_{22} \\ \epsilon_{12}\end{array}\right]=\left[\begin{array}{l}\epsilon_{1} \\ \epsilon_{2} \\ \epsilon_{6}\end{array}\right]$

For a given subcell $\Omega$, the mesh is tested to identify the elements inside or intersecting the domain, as shown Fig. 2. Considering each selected element $E_{i}^{\Omega}$, its intersected surface $S_{i}^{\Omega}\left(E_{i}^{\Omega}\right)$ is calculated through basic geometric operations.

The average estimation on subcell $\Omega$ for any spatial functions is expressed with a discrete weighted sum. For instance the average strain is given Eq. (10) and contains only surfaces and integration point values.

$\bar{\epsilon}^{\omega}=\frac{1}{\sum_{E_{i}^{\Omega}} S_{i}^{\Omega}\left(E_{i}^{\Omega}\right)} \sum_{E_{i}^{\Omega}} S_{i}^{\Omega}\left(E_{i}^{\Omega}\right) \epsilon\left(E_{i}^{\Omega}\right)$

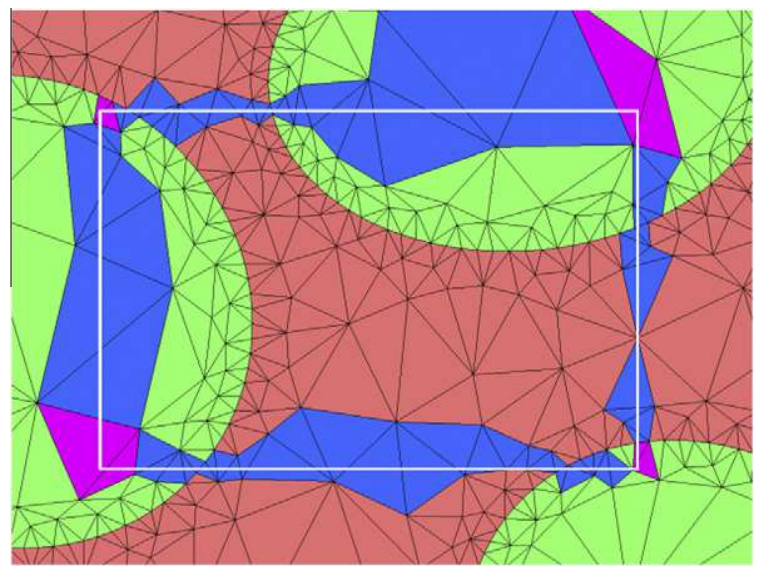

Fig. 2. Detail of heterogeneous meshing of fibres (green) and matrix (red) and identification of intersecting elements with one single boundary (blue) or a corner (purple). (For interpretation of the references to color in this figure legend, the reader is referred to the web version of this article.)
Perturbation fields are then computed.

The effective stiffness tensor is assessed with a discrete sum on cell $\Omega$, as expressed in Eq. (11).

$C_{e f f}^{\Omega}=\frac{1}{|\Omega|} \sum_{\Omega} C\left(E_{i}^{\Omega}\right)+C\left(E_{i}^{\Omega}\right) M_{i}^{\Omega}$

With the same method applied to stress fields and concentration tensors we obtain the effective compliance. In the two cases, we need to know the local tensors to explain the effective properties.

Three unit loading cases subscripted I, II and III are applied to the heterogeneous cell by kinematic conditions on the boundaries: (i) traction along $\overrightarrow{e_{1}}$ direction with global strain $E^{I}$, (ii) traction along $\overrightarrow{e_{2}}$ with global strain $E^{I I}$ and (iii) a plane shearing with global strain $E^{I I I}$. These three cases constitute a complete basis of loadings in the three dimensions loading linear space.

By convenience, a matrix representation of the local fields for the three basic loading cases is adopted for further identification processes. Local strain total matrix is given Eq. (12).

$\epsilon_{[t o t]}=\left[\begin{array}{lll}\epsilon^{I} & \epsilon^{I I} & \epsilon^{I I I}\end{array}\right]=\left[\begin{array}{ccc}\epsilon_{1}^{I} & \epsilon_{1}^{I I} & \epsilon_{1}^{I I I} \\ \epsilon_{2}^{I} & \epsilon_{2}^{I I} & \epsilon_{2}^{I I I} \\ \epsilon_{6}^{I} & \epsilon_{6}^{I I} & \epsilon_{6}^{I I I}\end{array}\right]$

Perturbation fields are concatenated this way and the localization tensor is identified as explained in Eq. (13).

$M_{i}^{\Omega}=\tilde{\epsilon}_{i[t o t]}^{\Omega} \bar{\epsilon}_{[t o t]}^{-1}$

In order to validate the numerical performance of this procedure, it is applied to a square periodic microstructure of fibres in matrix submitted to periodic loading conditions. This configuration is commonly used in multiscale modelling of composite materials. In our case, the simulation cell contains four fibres as seen on Fig. 3 to sample various locations of the homogenization subcell. The micro/meso energy balance is systematically verified with periodic fields, the average of perturbations product being equal to zero [9]. This is one reason why most of homogenization approach use periodic boundary conditions on RVEs.

The influence of mesh refinement parametrized by the number of nodes on edges was investigated: three simulation cells were build with 20,50 and 100 nodes on edges as seen Fig. 3. The homogenization subcells are squares, a quarter of the total cell

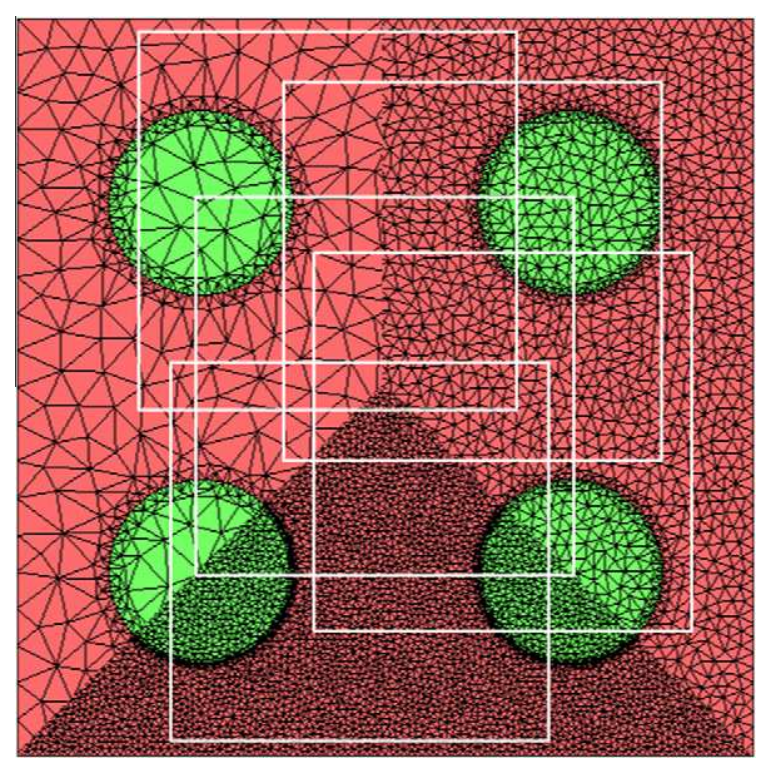

Fig. 3. Mesh refinement of the periodic cell with four fibres, 20, 50 and 100 nodes on boundaries and various homogenization subcells location (white squares). 
Table 1

Influence of meshing refinement on the estimation of effective properties.

\begin{tabular}{rlllll}
\hline$N_{\text {elts }}$ & $C_{11}$ & $C_{22}$ & $C_{12}$ & $C_{21}$ & $C_{66}[\mathrm{MPa}]$ \\
\hline 20 & 12777 & 12778 & 7893 & 7893 & 2153 \\
50 & 12839 & 12834 & 7871 & 7869 & 2174 \\
100 & 12789 & 12789 & 7788 & 7788 & 2158 \\
\hline
\end{tabular}

square, each containing one entire or splitted fibre and random locations were chosen.

As expected, for a given refinement, the effective stiffness is independent of subcell location. Behaviour tensors are symmetric, as shown in Table 1 . There is still a small variability depending on mesh refinement, inferior to $0.3 \%$ for $C_{i i}$ and $0.7 \%$ for $C_{i j}$. The method was performed with localization and concentration tensors and gave equal elastic properties.

The energy balance indicator is inferior to $1^{-3}$ for 20 nodes on edges, $1^{-4}$ for 50 nodes and $5^{-5}$ for 100 nodes. It remains satisfactory regardless to mesh refinement characteristics. The use of linear interpolation elements is not a limiting point. The numerical procedure is validated and can be confidently applied to sample homogenization of random heterogeneous media.

\section{Application to a LFRC with carbon fibres and epoxy matrix}

\subsection{Microscopic scale modelling of the heterogeneous ply}

The FE modelling is based on a transverse micrography of a ply, seen on Fig. 4(a). The contrast of the heterogeneities is balanced all over the image to perform a threshold on grey levels. Fibre sections, whose shape are actually quite irregular probably due to fabrication process, are fitted by ellipses and their geometric characteristics - centre, minor and major axes and orientation.

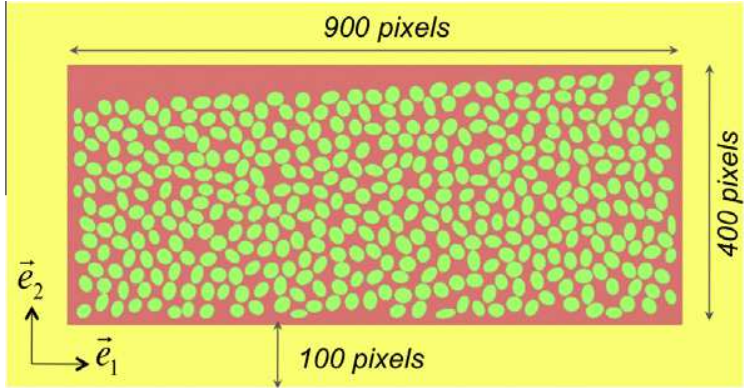

Fig. 5. Simulation cell: microstructure of fibres and matrix surrounded by a band of equivalent homogeneous medium and length scale in pixels.

These data are computed in a specifically developed meshing procedure to produce the FE mesh, as shown on Fig. 4(c).

A large part of a ply's heterogeneous microstructure, 0.073 $\times 0.171 \mathrm{~mm}^{2}$ equals $400 \times 900 \mathrm{pp}$, is modelled. It is surrounded with a band of Homogeneous Equivalent Medium (HEM) to reduce edge effects on nearby fibres, as shown on Fig. 5 as in the Embedded Cell Approach developed in [2] and applied in [19]. The average behaviour of the heterogeneous microstructure is estimated though an iterative procedure based on the balance of average stresses in the heterogeneous and homogeneous media.

Kinematic loading conditions are applied on the boundaries to simulate the three basic loading cases described in Section 3.1, under 2D plane strain hypothesis. Material properties correspond to a carbon fibres and epoxy matrix composite transversely to the fibers:

- fibres: $E_{f}=390 \mathrm{GPa}, v_{f}=0.35$,

- matrix: $E_{m}=4.5 \mathrm{GPa}, v_{m}=0.4$

- HEM: $E_{\text {HEM }}=15 \mathrm{GPa}, v_{\text {HEM }}=0.35$, a

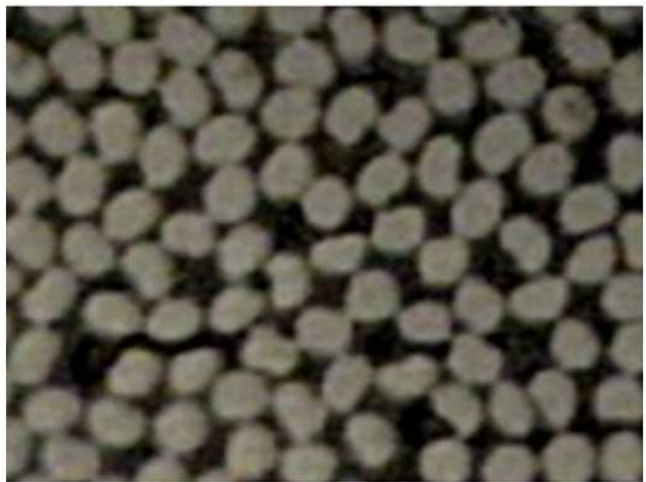

b





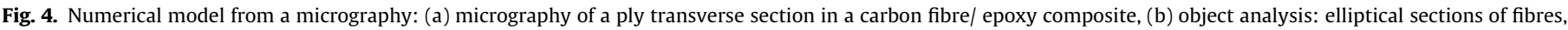
(c) mesh of the ply microstructure: fibres and matrix. 


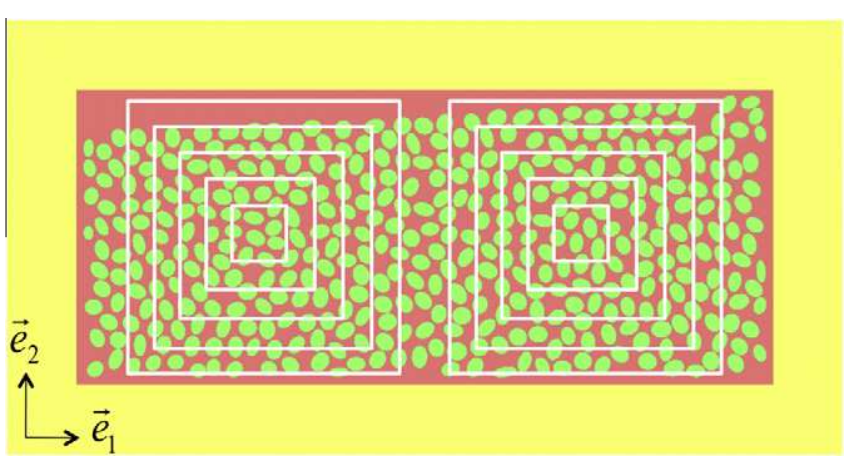

Fig. 6. Simulation cell: locations of two sets of variable size homogenization sampling cells.

\subsection{Influence of subsampling size on energy accuracy}

The first sampled set of subcells allows to investigate the influence of homogenization subcells size on the energy balance. As explained in [6], there is an intrinsic homogenization size under which the general theory presented does not verify Hill's energy balance. This minimal length concept was investigated on periodic homogenization and is assumed to be transposable to our work on non periodic homogenization. Two locations are chosen to generate two sets of variable size concentric square subcells, as depicted on Fig. 6.

The strong variations of subcells effective properties versus the homogenization size are shown on Fig. 7. Stiffness increases from small to medium subcells: smaller ones might be centred on a matrix zone. It decreases from medium to high sizes: the biggest cells contain a higher ratio of matrix as they approach the heterogeneous medium boundaries. As expected, the energy balance is better on bigger cells, as shown on Fig. 8.

The negative values of the average of perturbations product are pointed out. Indeed it is not a regular elastic strain energy which would be positive. It can be seen as a energy offset between scales, which can be either positive or negative - or zero. It is caused by the existence of elements in which perturbation strain and stress are of opposite signs.

For the following analyses the energy unbalance is supposed to be acceptable for a subcell size larger than $100 \mathrm{p}$. With a given size we can investigate the variability of effective properties and the influence of sampling parameters on the statistical moments.

\subsection{Statistical distributions of effective properties}

Various spatial subsampling schames are performed to investigate their effect on statistical characteristics of mechanical properties variability. It is indeed necessary to control the statistical inference as the characterized distribution functions will be used in mesoscopic simulations.

\subsubsection{Regular pattern subsampling}

Two realizations of subcells sampling with two different sizes are presented: $200 \mathrm{p}$ equals $0.036 \mathrm{~mm}$ - half the height of the ply - and 300 p equals $0.056 \mathrm{~mm}$. A regular spatial scheme is chosen for both sampling sizes: the cells are repartitioned every ten pixels in both $\overrightarrow{e_{1}}$ and $\overrightarrow{e_{2}}$ directions.

These sizes are larger than the limit size validated in the previous section to verify the inter scale energy equivalence. It is confirmed by the relative energy error indicator inferior to $1.5 \%$. The dispersion are presented in function of the fibre volume fraction on Fig. 9(a) and (b). It shows an important variability of stiffness for a given volume fraction, around $10 \%$ which is far higher than
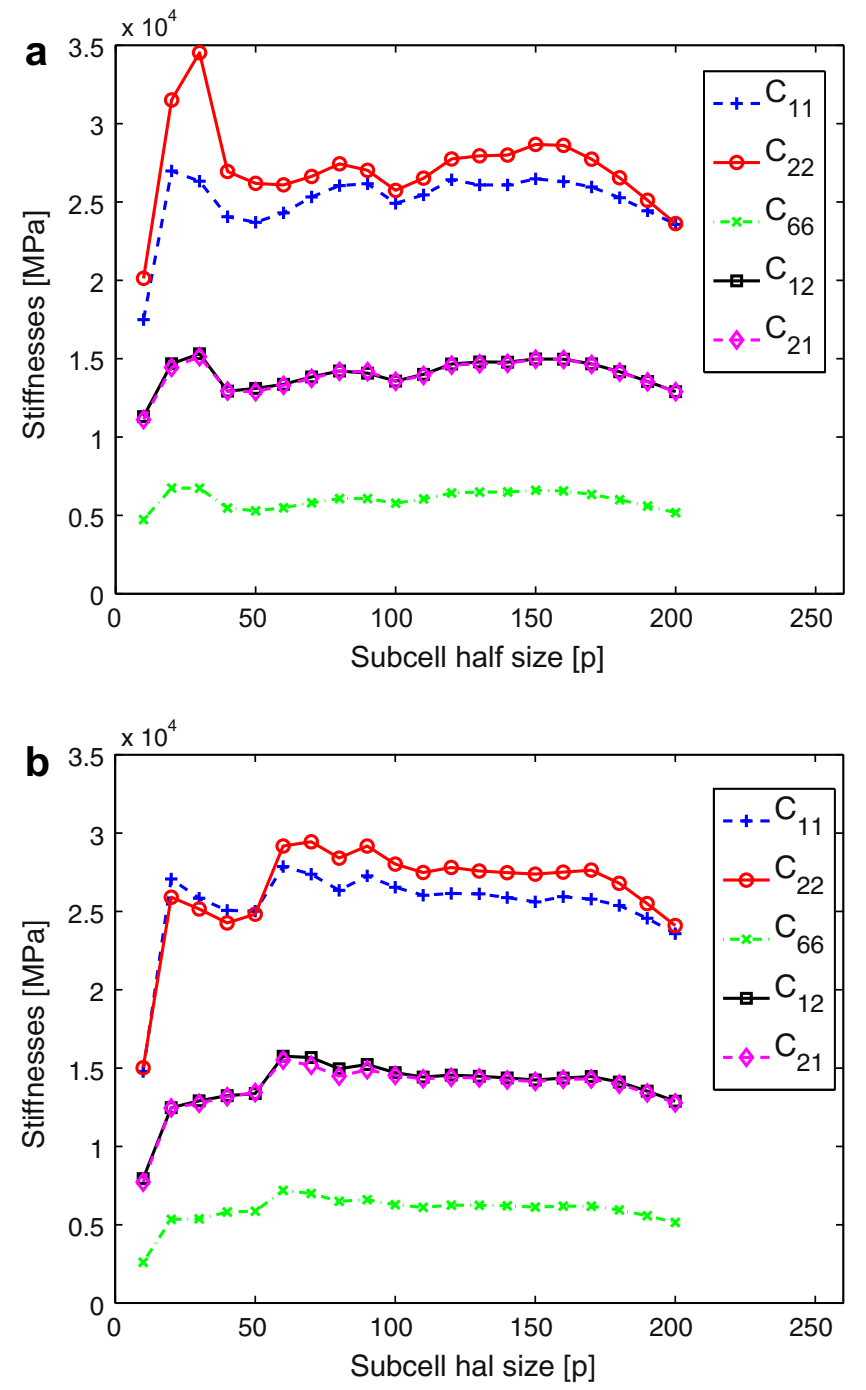

Fig. 7. Variations of effective properties on the two sets versus cell size.

th $1 \%$ value obtained in [4] with non-cutte fibres on edges. This result confirms those obtained in [1].

A linear regression using least square method gives approximations of $C_{11}$ and $C_{22}$ in function of fibre volumic fraction $V_{f}$ for 200 pp and 300 pp subcells Eqs. (14), (15).

$$
\begin{array}{ll}
200 \mathrm{pp}: C_{11}=39577 V_{f}+4513, & C_{22}=62354 V_{f}-5880 \\
300 \mathrm{pp}: C_{11}=36510 V_{f}+6141, & C_{22}=61144 V_{f}-5183
\end{array}
$$

Global effective properties can be evaluated applying the nonperiodic geometry homogenization procedure to the largest subcells as possible taken in the ply, around $400 \mathrm{p}$ wide. They are listed Table 2. They fit correctly with Eqs. (14), (15) which can be considered as apropriate estimations of average stiffnesses in regard to the volume fraction.

In order to model the variability in FE meshes at meso scale, we need to statistically characterise the dispersions. The identification of normal distribution parameters (average $\mu$ and standard deviation $\sigma$ ) for $C_{11}$ stiffness resulted in:

- for 200 pp subsample: $\mu=24986$ and $\sigma=1261$,

- for 300 pp subsample: $\mu=25099$ and $\sigma=742$.

It clearly shows, as expected, that dispersions on volume fractions and equivalent properties are smaller with the larger subcells. For 

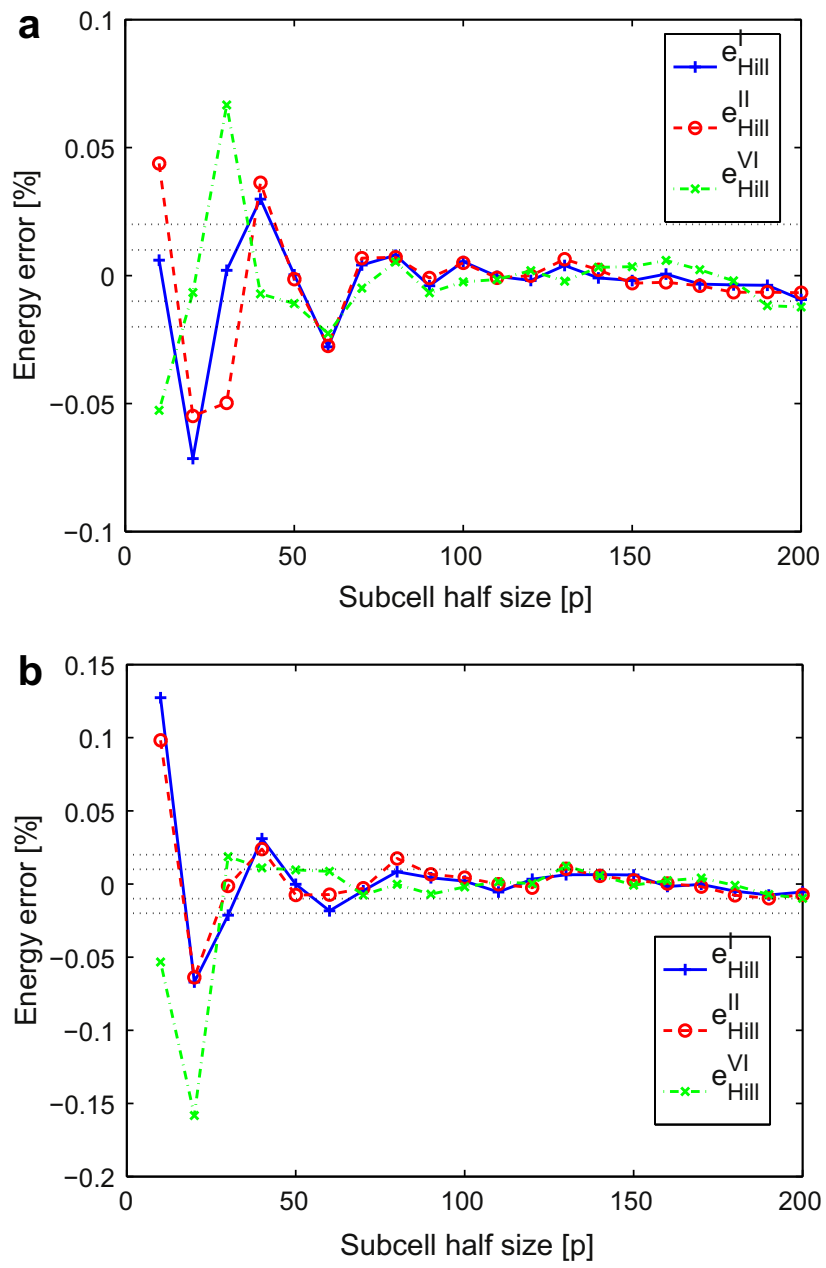

Fig. 8. Energy balance indicator on the two sets versus cell size, dotted lines at $\pm 1 \%$ and $\pm 2 \%$.

example, for a given volume fraction, dispersion of effective properties is wider for smaller subcells. It is also clear that microstructure is unbalanced and the material is stiffer in direction $\overrightarrow{e_{2}}$.

\subsubsection{Random subsampling}

Several independent random subsamplings have been computed varying the size of the sample $\mathrm{N}_{s b_{s} a m p}$ : 25 (three times), 50 (two times), 300 and 500; they constitute a whole global sample of 975 subcells. A uniform distribution was used to generate the positions $(x, y)$ of subcells center. Normal dispersion functions for $C_{11}$ stiffness have been identified, the average and standard deviations are liste Table 3 . The estimation of average $\mu$ is accurate with low size subsampling but not for standard deviation $\sigma$.

To refine these results, the intern combinations in the 500 subsamples population has been explored in details. For a $N$ elements population, subpopulations of $p$ elements $(p \in[1, N])$ can be extracted with a couting of $A_{p}^{n}$ possibilities. It is possible to identify $p$ subpopulation with highest or lowest stiffness, which defines bounds for average value $\mu$. For each $p$ value, 15 combinations have been randomed, their statistical parameters are given Fig. 10 .

Low size subpopulation gives rapidly an accurate estimation of average value $\mu$ but standard deviation needs high size subsampling. One can notice that standard deviation for highest or lowest stiffness subpopulation are not bounds for random subsampling.
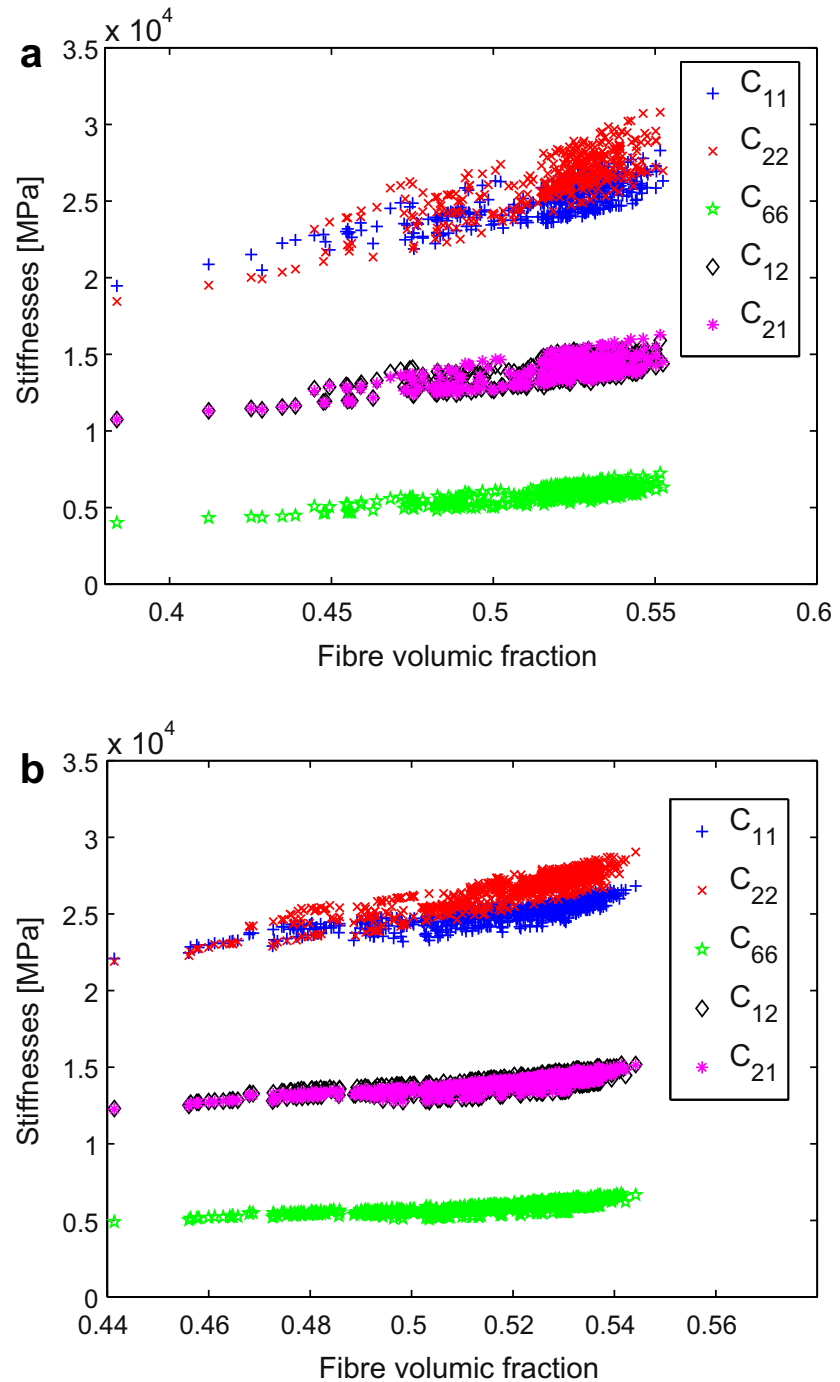

Fig. 9. Variability of $C_{i i}$ versus fibre volume fraction with linear interpolation of average stiffnesses for $C_{11}$ and $C_{22}$ : (a) $200 \times 200$ pp subcells and (b) $300 \times 300$ pp subcells.

\subsubsection{Influence of sampling scheme: statistical inference}

It appears from the two former paragraphs that the subsampling scheme influences the statistical characterization of elastic properties. To highlight this effet, normal variability functions are identified on various subsamples:

- two times 10 subcells with $0 \%$ overlapping,

- 24 subcells with $50 \%$ overlapping,

- 396 subcells with 95\% overlapping.

The distribution functions of stiffness $C_{11}$ for various overlapping on regular spatial schemes are presented on Fig. 11 for the $200 \times 200$ pp subcells. This effect is caused by the geostatistical representativeness of subcells in various sampled identification data sets. For example non overlapping subcells contain more matrix as they touch boundaries than cells in the center of the microstructure. This effect underlines the issue of samples representativeness and its implications on the parameters values of the dispersion functions as it was also underlined in the previous section for random subsampling. Applications of sampling have been studied for geomorphological properties of heterogeneous media. 
Table 2

$C_{11}$ and $C_{22}$ stiffnesses versus $V_{f}$ for very large subcells.

\begin{tabular}{lll}
\hline$V_{f}$ & $C_{11}$ & $C_{22}[\mathrm{MPa}]$ \\
\hline 0.4827 & 23448 & 24417 \\
0.4980 & 23931 & 25184 \\
0.5067 & 24430 & 25559 \\
0.5168 & 24811 & 26254 \\
\hline
\end{tabular}

Table 3

Identification of normal distribution statistical moments.

\begin{tabular}{lllllllll}
\hline$N_{\text {ss-ech }}$ & 25 & & & 50 & & 300 & 500 & 975 \\
\hline$\mu$ & 25296 & 25121 & 25394 & 25132 & 25202 & 25132 & 25234 & 25223 \\
$\sigma$ & 975 & 944 & 1234 & 1206 & 998 & 1115 & 1045 & 1072 \\
\hline
\end{tabular}
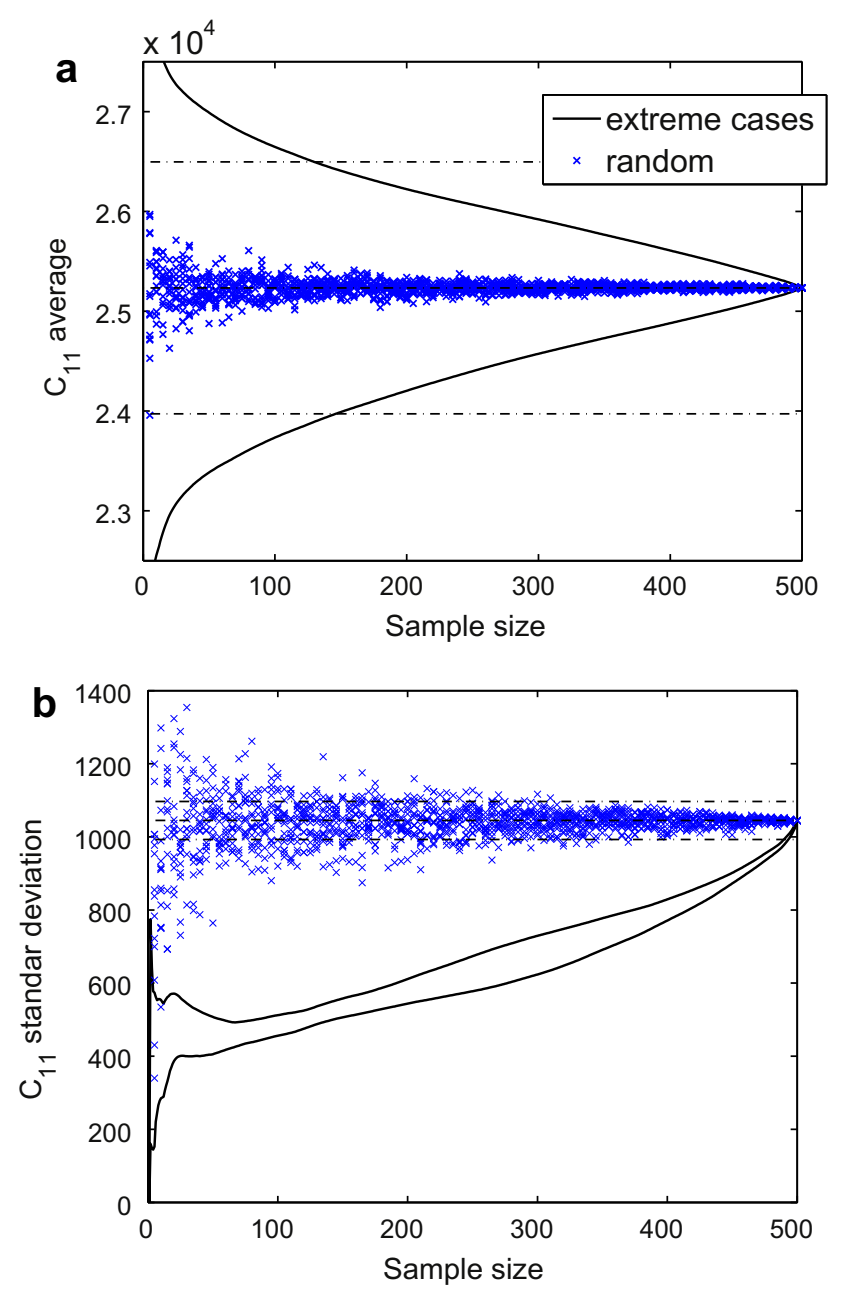

Fig. 10. Identification of statistical moments for subsamples of the 500 population; parameters for the least or most stiff subsamples traced in black lines, dotted lines stands for $\pm 5 \%$ difference with average values.

The problem here for the non-overlapping sampling scheme is the small numbers of subcells that can be extracted from the entire population is not morphologically representative of the entire ply and as a consequence the effective properties aren't either. For overlapping subcells, it appears that stiffness increases with the overlapping rate. With a $90 \%$ rate, the proportion of subcells with lower fibre volume fraction located on the edges of the ply is higher in the entire population, it can explain the lower values.

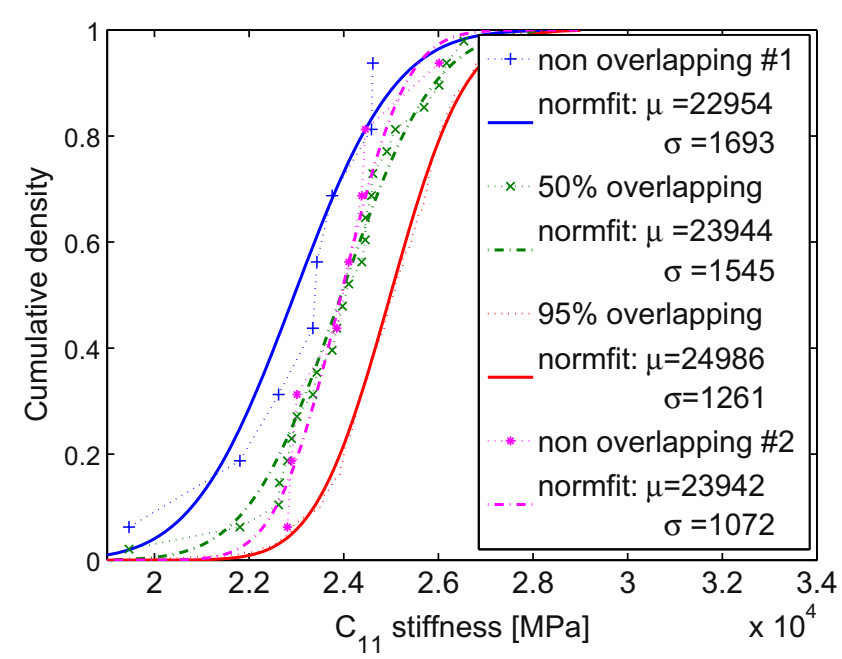

Fig. 11. Geostatistical variability of $C_{11}$ microscopic variability depending on the overlapping scheme fitted with Normal dispersion functions: mean and variance.

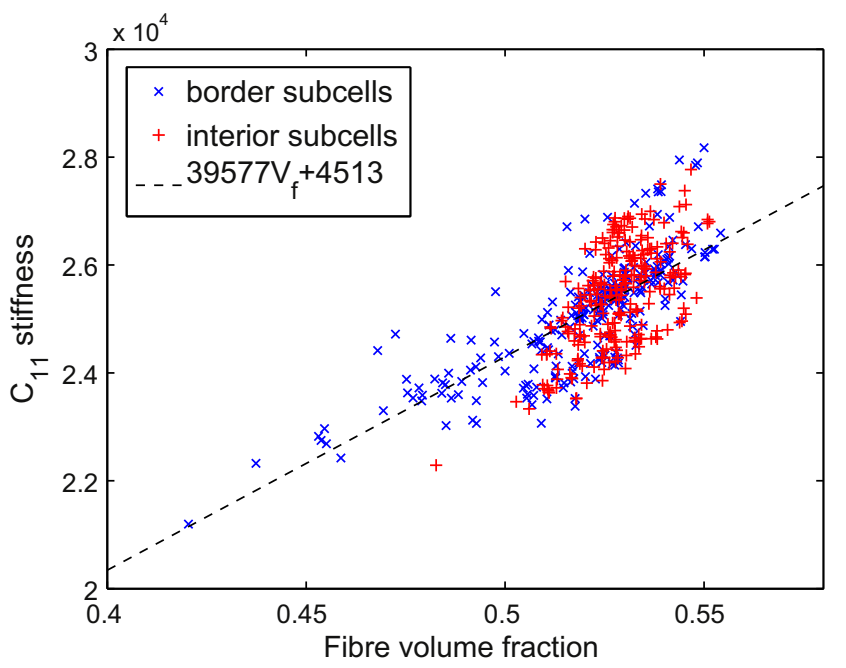

Fig. 12. $C_{11}$ stiffness of cells located close to the borders in blue and in the central part in red. (For interpretation of the references to color in this figure legend, the reader is referred to the web version of this article.)

In comparison with Babuska's work [1], high variability was obtained as well for subcells. But it has been highlighted that spatial regular pattern is limited as it is not representative enough of the entire microstructure and introduces a high statistical bias in distribution function identification.

It is shown in previous section that a random spatial scheme avoids a bad identification. Statistical average $\mu$ is well identified with low size subsamples, around 150 , and standard deviation $\sigma$ needs high size subsamples, around 400 . It is supposed that overrepresentation of subcells on border are responsible for bad identification on regular spatial schemes. The population of 500 subsamples has been split in two halfs with close to border and central subcells. The dispersion for $C_{11}$ are presented Fig. 12. Except for very low fibre volume fraction subcells, dispersion seems equal. Though, it shows that the use of the statistical distribution functions needs to be completed with a morphological analysis, for exemple at first order the volume fraction. 


\section{Conclusion and outlook}

An original subsampling procedure has been developed at postprocessing level for numerical homogenization on non-periodic geometry. It is applied to obtain the dispersion of LFRC effective elastic properties at mesoscopic scale. The microstructure of a transverse section ply of a LFRC is modeled and meshed to achieve FEA simulations. Various samples of subcells of the heterogeneous medium are homogenized to obtain effective properties.

A limit size to achieve the microscopic - mesoscopic energy balance with non periodic geometry an natural boundary conditions is highlighted. For a valid subscells size, the dispersion functions of stiffness at mesoscopic scale are identified. They depict a strong variability for given fibre volume fractions. It is assumed to be a consequence of the numerous random configurations of fibre cuts on subcells' boundaries.

This study also reveals the statistical inference induced by the chosen sampling spatial scheme on the effective properties variability functions confronting various results obtained with regular and random spatial scheme subsamplings. Complementary investigations could be carried out on this subject to improve reliability and representativeness of samplings mainly with morphological analysis of microsructure.

In further works, the statistical dispersions obtained here will be used to randomly assign effective properties to homogeneous subcells constituting a ply at mesoscale. This work will set for a structural analysis at mesoscale taking into account variability induced by microscale and will be presented in future publications. Another research orientation consits in modelling damage and failures and investigate its dependance on the microstructure properties.

\section{References}

[1] I. Babuska, B. Andersson, P.J. Smith, K. Levin, Damage analysis of fiber composites. Part I: Statistical analysis on fiber scale, Comput. Methods Appl. Mech. Engrg. 172 (1999) 27-77.
[2] J.R. Brockenbrough, S. Suresh, H.A. Wienecke, Deformation of metal-matrix composites with continuous fibers: geometrical effects of fiber distribution and shape, Acta Metall. Mater. 39-5 (1991) 735-752.

[3] G. Cailletaud, S. Forest, D. Jeulin, F. Feyel, I. Gallier, V. Mounoury, S. Quilici, Some elements of microstructural mechanics, Comput. Mater. Sci. 27 (2003) 351-374.

[4] R. Chermaneanu, L. Guillaumat, F. Dau, Uncertainties propagation in composite materials, in: Proceedings of Journées Nationales sur les Composites 16 (JNC16), Toulouse, France, 2009.

[5] F. Dau, A. Alzina, L. Guillaumat, Uncertainties propagations of composite mechanical properties from micro to macro scale, in: Proceedings of the thirteenth European Conference on Composite Materials (ECCM13), Stockholm, Sweden, 2008.

[6] S. Forest, F. Pradel, K. Sab, Asymptotic analysis of heterogeneous cosserat media, Int. J. Solids Struct. 38 (2001) 4585-4608.

[7] R. Hill, Elastic properties of reinforced soils: some technical principles, J. Mech. Phys. Solids 11 (1963) 357-372.

[8] S.J. Hollister, J.M. Brennan, N. Kikuchi, A homogenization sampling procedure for calculating trabecular bon effective stiffness and tissue level stress, J. Biomech. 27 (4) (1994) 433-444.

[9] S.J. Hollister, N. Kikuchi, A comparision of homogenization and standard mechanics analyses for periodic porous composites, Comput. Mech. 10 (1992) 73-95.

[10] C. Huet, Application of variational concepts to size effects in elastic heterogeneous bodies, J. Mech. Phys. Solids 38 (6) (1990) 813-841.

[11] T. Kanit, S. Forest, I. Galliet, V. Mounoury, D. Jeulin, Determination of the size of the representative volume element for random composites: statistical and numerical approach, Int. J. Solids Struct. 40 (2003) 3647-3679.

[12] S.N. Lahiri, M.S. Kaser, N. Cressie, N.J. Hsu, Prediction of spatial cumulative distribution functions using subsampling, J. Am. Stat. Assoc. 94 (445) (1999) 86-110.

[13] D.N. Politis, M. Sherman, Moment estimation for statistics from marked point processes, Journal of the Royal Statistical Society B 63 (2001) 261-275. part 2.

[14] B.D. Ripley, Statistical inference for spatial process, Cambridge University Press, 1988.

[15] K. Sab, On the homogenization and the simulation of random materials, Eur. J. Mech. A. Solids 11 (5) (1992) 585-607.

[16] S. Sakata, F. Ashida, M. Zako, Kriging-based approximate stochastic homogenization analysis for composite materials, Comput. Methods Appl. Mech. Engrg. 197 (2008) 1953-1964.

[17] in: E. Sanchez-Palencia, A. Zaoui, (eds.), Lecture Notes in Physics, vol. 272, Springer-Verlag, Berlin.

[18] S. Shkoller, An approximate homogenization scheme for non-periodic materials, Comput. Math. Appl. 33 (4) (1997) 15-34.

[19] D. Trias, J. Costa, A. Turon, J.E. Hurtado, Determination of the critical size of a statistical representative volume element (srve) for carbon reinforced polymers, Acta Mater. 54 (2006) 3471-3484 